\title{
Asymptotics of solution of a boundary value problem for a singularly perturbed quasilinear one-characteristic equation
}

\author{
Mahir M. Sabzaliev ${ }^{\star}$ • Mahbuba E. Kerimova
}

Received: 08.07.2019 / Revised: 10.03.2020 / Accepted: 08.04.2020

\begin{abstract}
A complete asymptotics of the solution of a boundary value problem on a rectangle is constructed for one-characteristic nonclassic type differential equation of third order, degenerating into nonlinear hyperbolic equation. The remainder term is estimated.
\end{abstract}

Keywords. asymptotics, boundary layer type function, remainder term.

Mathematics Subject Classification (2010): 35B25, 35C20, 35G30

\section{Introduction and problem statement}

When some studying real phenomena with nonuniform passages from one physical characterstics to other ones, it is necessary to study singularly perturbed boundary value problems. The problem of dynamics of solids with cavities containing viscous fluid are the examples for such problems. Singularly perturebed boundary value problems have attracted attention of such prominent scientsist as N.N. Bogolyubov, Yu.A. Mitropolskii, V. Vazov, A.N. Tikhonov, L.S. Pontryagin, K. Friedrichs, M.I. Vishik, L.A. Lusternik and others. In [1], [2] by M.I. Vishik and L.A. Lusternik were essential development of theory of singularly perturbed problems for partial equations. The asymptotic method developed by them (at present it is called the Vishik-Lusternik method) is widespread. The Vishik-Lusternik method for constructing asymptotics in a small parameter of solutions of boundary value problems for linear differential equations is carried on other classes of nonlinear differential equations as well. However, study of nonlinear singularly perturbed boundary value problems with this method is accompanied with bulky calculations. The study of every nonlinear equation requires from the researcher a special approach. It should be noted that the majority of the investigated perturbed partial differential equations referred to one of three classic types.

In [1] M.I. Vishik and L.A. Lusternik introduced the so called one-characteristic linear equations that are not classical equations. They called the $2 k+1$ odd order equation of the form, $A_{1}\left(A_{2 k} u\right)+B_{2 k} u=f$ one-characteristic if $A_{1}$ is a first order operator, $A_{2 k}$ is an

\footnotetext{
* Corresponding author

M.M. Sabzaliev

Baku Business University, Baku, Azerbaijan

E-mail: sabzalievm@mail.ru

M.E. Kerimova

Azerbaijan State Oil and Industry University, Baku, Azerbaijan

E-mail: maxbuba3773@mail.ru
} 
elliptic operator of order $2 k$, while $B_{2 k}$ is any differential operator of order at most $2 k$. In the paper [1], they studied mutual degenerations of one-characteristic and elliptic equations. In [3]-[5], the boundary value problems stated for a third order one-characteristic differential equation degenerated into a second order parabolic equation in a rectangular domain, finite half-strip and infinite strip are considered and complete asymptotics of the solutions of these problems with respect to a small parameter are constructed. In this paper, we consider a boundary value problem stated in a rectangular domain for an one-characteristic differential equation degenerating into a first order nonlinear hyperbolic one.

In the rectangular domain $D=\{(x, y) \mid 0 \leq x \leq 1,0 \leq y \leq 1\}$ we consider the following boundary value problem:

$$
\begin{gathered}
L_{\varepsilon} u \equiv \varepsilon^{2} \frac{\partial}{\partial x}(\Delta u)-\varepsilon \Delta u+\frac{\partial u}{\partial x}+\frac{\partial u}{\partial y}+F(x, y, u)=0, \\
\left.u\right|_{x=0}=\left.u\right|_{x=1}=0,\left.\quad \frac{\partial u}{\partial x}\right|_{x=1}=0,0 \leq y \leq 1, \\
\left.u\right|_{y=0}=\left.u\right|_{y=1}=0,0 \leq x \leq 1 .
\end{gathered}
$$

where $\varepsilon>0$ is a small parameter, $\Delta \equiv \frac{\partial^{2}}{\partial x^{2}}+\frac{\partial^{2}}{\partial y^{2}}$ is a Laplace operator, $F(x, y, u)$ is a given function satisfying the following conditions:

$$
\begin{gathered}
F(x, y, 0) \not \equiv 0,(x, y) \in D, \\
\frac{\partial F(x, y, u)}{\partial u} \geq \gamma^{2}>0,(x, y, u) \in D \backslash\{(x, y) \in D \mid x=y\} \times(-\infty,+\infty) .
\end{gathered}
$$

Note that the function $F(x, y, u)$ may depend on the variable $u$ both linearly and nonlinearly. In the case of linear dependence we consider

$$
F(x, y, u)=a(x, y) u-f(x, y), a(x, y) \geq \gamma^{2},(x, y) \in D .
$$

The goal of the paper to construct asymptotic expansion of the solution of problem (1.1)-(1.3) with respect to a small parameter. For that we conduct iterative processes.

\section{Conducting the first iterative process}

In the first iterative process, the approximate solution of the equation

$$
L_{\varepsilon} W=0\left(\varepsilon^{n+1}\right)
$$

is sought in the form:

$$
W=W_{0}+\varepsilon W_{1}+\ldots+\varepsilon^{n} W_{n}=W_{0}+\delta W .
$$

Here we denote $\delta W=\sum_{i=1}^{n} \varepsilon^{i} W_{i}$. Substituting the expression of $W$ in (2.2) in the equality (2.1), we write the expansion of the function $F\left(x, y, W_{0}+\delta W\right)$ at the point $\left(x, y, W_{0}\right)$ in Taylor formula, after making grouping with respect to the same order powers of $\varepsilon$, for determining $W_{i}(x, y) ; i=0,1, \ldots, n$ we obtain the following equalities:

$$
\begin{gathered}
\frac{\partial W_{0}}{\partial x}+\frac{\partial W_{0}}{\partial y}+F\left(x, y, W_{0}\right)=0, \\
\frac{\partial W_{j}}{\partial x}+\frac{\partial W_{j}}{\partial y}+\frac{\partial F\left(x, y, W_{0}\right)}{\partial W_{0}} W_{j}=f_{j}\left(W_{0}, W_{1}, \ldots, W_{j-1}\right) ; j=1,2, \ldots, n .
\end{gathered}
$$


Here $f_{j}\left(W_{0}, W_{1}, \ldots, W_{j-1}\right)$ denote the known functions expressed by the functions $W_{0}, W_{1}$, $\ldots, W_{j-1}$, themselves, their first, second and third order derivatives. We can write their explicit expressions, but their formulas are very bulky. Below we give explicit expressions of the functions $f_{1}$ and $f_{2}$ :

$$
\begin{gathered}
f_{1}\left(W_{0}\right)=\Delta W_{0}, \\
f_{2}\left(W_{0}, W_{1}\right)=\Delta W_{1}-\frac{\partial}{\partial x}\left(\Delta W_{0}\right)-\frac{1}{2 !} \frac{\partial^{2} F\left(x, y, W_{0}\right)}{\partial W_{0}^{2}} W_{1}^{2} .
\end{gathered}
$$

As can be seen, equality (2.3) is nonlinear, equalities (2.4) are linear. We will find the solutions of equations (2.3), (2.4) satisfying the boundary conditions

$$
\left.W_{i}\right|_{x=0}=0,0 \leq y \leq 1 ;\left.W_{i}\right|_{y=0}=0,0 \leq x \leq 1 ; i=0,1, \ldots, n .
$$

Thus, from (2.3) and for $i=0$ from (2.7) we get that the function $W_{0}(x, y)$ is the solution of the boundary value problem

$$
\frac{\partial W_{0}}{\partial x}+\frac{\partial W_{0}}{\partial y}+F\left(x, y, W_{0}\right)=0 ;\left.W_{0}\right|_{x=0}=0,\left.W_{0}\right|_{y=0}=0 .
$$

The problem (2.8) is said to be a degenerate problem corresponding to problem (1.1), (1.3). When the function $F(x, y, u)$ is linearly dependent on $u$ (see (1.6)) the problem corresponding to degenerate problem (1.1)-(1.3) is in the form

$$
\frac{\partial W_{0}}{\partial x}+\frac{\partial W_{0}}{\partial y}+a(x, y) W_{0}=f(x, y) ;\left.W_{0}\right|_{x=0}=0,\left.W_{0}\right|_{y=0}=0
$$

Researches show that even if the $f(x, y)$ is a rather smooth function, the solution of problem (2.9) has a singularity on the line $y=x$ being the bisector of the first quarter. But the right hand side of each of the equations (2.4) where the functions $W_{1}, W_{2}, \ldots, W_{n}$ will be defined, contain the preceding functions themselves and their derivatives. Therefore, to complete the first iterative process, the function $W_{0}(x, y)$ should be a rather smooth function.

The following proposition was proved in [6].

Lemma 2.1 Assume that when $F(x, y, u) \in C^{k}(D \times(-\infty,+\infty))$ and the function $F(x, y, u)$ is linearly dependent on the variable $u$ satisfies the condition

$$
\left.\frac{\partial^{i} f(x, y)}{\partial x^{i_{1}} \partial y^{i_{2}}}\right|_{x=y}=0 ; i=i_{1}+i_{2} ; i=0,1, \ldots, k ; 0 \leq x \leq 1
$$

when is linearly in dependent of the variable $u$, satisfies the conditions

$$
\begin{gathered}
\left.F(x, y, u)\right|_{x=y}=0 ; 0 \leq x \leq 1, u \in(-\infty,+\infty), \\
\left.\frac{\partial^{i} F(x, y, 0)}{\partial x^{i_{1}} \partial y^{i_{2}} \partial u^{i_{3}}}\right|_{x=y}=0 ; 0 \leq x \leq 1 ; i=i_{1}+i_{2}+i_{3} ; i=1,2, \ldots, k .
\end{gathered}
$$

Here $k$ is an arbitrary natural number. Then problem (2.8) has a unique solution and this solution satisfies the conditions $W_{0}(x, y) \in C^{k}(D)$ and

$$
\left.\frac{\partial^{i} W_{0}(x, y)}{\partial x^{i_{1}} \partial y^{i_{2}}}\right|_{x=y}=0 ; i=i_{1}+i_{2} ; i=0,1, \ldots, k ; 0 \leq x \leq 1 .
$$

We do not give here the proof of Lemma 2.1. One can see the proof process in the proof of theorem 1 [6] p. 108. We should only note that when the function $F(x, y, u)$ is linearly 
dependent on the variable $u$, the solution of the degenerate problem, i.e. the solution of problem (2.9) is determined by the formula

$$
W_{0}=\left\{\begin{array}{l}
\int_{0}^{x} f(\xi, \xi+y-x) \exp \left[-\int_{\xi}^{x} a(\tau, \tau+y-x) d \tau\right] d \xi, 0 \leq x<y \leq 1, \\
\int_{0}^{y} f(x-y+\xi, \xi) \exp \left[-\int_{\xi}^{y} a(x-y+\xi, \xi) d \tau\right] d \xi, 0 \leq y<x \leq 1 .
\end{array}\right.
$$

In this case, when the condition (2.10) is satisfied, using the formula (2.14) one can easily prove that condition (2.13) is satisfied.

When the function $F(x, y, u)$ is linearly independent of the variable $u$, the fulfilment of condition (2.13) is proved by using the nonlinear integral equation

$$
W_{0}(x, y)=\left\{\begin{array}{l}
-\int_{0}^{x} F\left(\tau, \tau+y-x, W_{0}(\tau, \tau+y-x)\right), 0 \leq x<y \leq 1, \\
-\int_{0}^{y} F\left(x-y+\tau, \tau, W_{0}(x-y+\tau, \tau)\right), 0 \leq y<x \leq 1
\end{array}\right.
$$

that is equivalent to boundary value problem (2.8).

So, we determined the function $W_{0}(x, y)$ being the solution of problem (2.5). For $j=1$ from (2.4) for $i=1$ from (2.7) we get that $W_{1}(x, y)$ is the solution of the following boundary value problem:

$$
\frac{\partial W_{1}}{\partial x}+\frac{\partial W_{1}}{\partial y}+a(x, y) W_{1}=\Delta W_{0} ;\left.W_{1}\right|_{x=0}=0,\left.W_{1}\right|_{y=0}=0 .
$$

Problem (2.15) is a boundary value problem of type (2.9). The only difference is that in the right hand side of the equation $\Delta W_{0}$ stands instead of $f(x, y)$. According to Lemma 2.1, in (2.15) the function in the right side of the equation $W_{1}$ and its all derivatives to the $k-2$ -order (including $k$-2 order) become zero for $x=y$. Therefore, lemma 2.1 can be applied to boundary value problem (2.15) as well. Continuing the process in such a way, we determine all the functions $W_{0}, W_{1}, \ldots, W_{n}$ in the right hand side of equality (2.2). Simple calculations show that for completing the first iterative process and then estimating the remainder term, for acting on the function $W=\sum_{i=0}^{n} \varepsilon^{i} W_{i}$ by the operator $L_{\varepsilon}$ we must take the natural number $k$ in the condition of lemma 2.1 as $k=2 n+3$.

Thus, we constructed such a function $W=\sum_{i=0}^{n} \varepsilon^{i} W_{i}$ that this function is an approximate solution of differential equation (1.1). From (2.7) and (2.2) we have that the constructed function $W$ satisfies the boundary conditions

$$
\left.W\right|_{x=0}=0,0 \leq y \leq 1 ;\left.W\right|_{y=0}=00 \leq x \leq 1 .
$$

But the function $W$ might not satisfy the boundary condition on $x=1$ in (1.2) and the boundary condition on $y=1$ in (2.3). To ensure satisfaction of these missing boundary conditions we must construct boundary layer type functions near the boundaries $S_{1}=\{(x, y) \mid x=1,0 \leq y \leq 1\}$ and $S_{2}==\{(x, y) \mid 0 \leq x \leq 1, y=1\}$.

For constructing these boundary layer functions we should construct iterative processes. In order to conduct iterative processes at first must write new expansions of the operator $L_{\varepsilon}$ near $S_{1}$ and $S_{2}$ with respect to a small parameter. 


\section{Conducting iterative processes for constructing boundary layer type functions}

Replace the coordinates near $S_{1}$ by the formulas $1-x=\varepsilon \tau, y=y$. Assume that the functions $r_{j}(\tau, y) ; j=0,1, \ldots, n+1$ are rather smooth functions determined in the domain $[0,+\infty) \times[0,1]$. We accept the following denotation:

$$
r=\sum_{j=0}^{n+1} \varepsilon^{j} r_{j}(\tau, y)=r_{0}+\delta r .
$$

Here $\delta r$ denotes $\delta r=\varepsilon r_{1}+\varepsilon^{2} r_{2}+\ldots+\varepsilon^{n+1} r_{n+1}$. Taking into account the above substitution in the expansion (1.1) of the operator $L_{\varepsilon}$, we expand the function $F\left(1-\varepsilon \tau, y, r_{0}+\delta r\right)$ at the point $\left(1, y, r_{0}\right)$ in Taylor formula, making grouping with respect to the same powers of $\varepsilon$, we get the following expansion for $L_{\varepsilon}\left(\sum_{j=0}^{n+1} \varepsilon^{j} r_{j}\right)$ :

$$
\begin{gathered}
L_{\varepsilon, 1} r \equiv L_{\varepsilon, 1}\left(\sum_{j=0}^{n+1} \varepsilon^{j} r_{j}\right) \equiv \varepsilon^{-1}\left\{-\left(\frac{\partial^{3} r_{0}}{\partial \tau^{3}}+\frac{\partial^{2} r_{0}}{\partial \tau^{2}}+\frac{\partial r_{0}}{\partial \tau}\right)\right. \\
+\varepsilon\left[-\left(\frac{\partial^{3} r_{1}}{\partial \tau^{3}}+\frac{\partial^{2} r_{1}}{\partial \tau^{2}}+\frac{\partial r_{1}}{\partial \tau}\right)+\frac{\partial r_{0}}{\partial y}+F\left(1, y, r_{0}\right)\right] \\
+\sum_{s=2}^{n+1} \varepsilon^{s}\left[-\left(\frac{\partial^{3} r_{s}}{\partial \tau^{3}}+\frac{\partial^{2} r_{s}}{\partial \tau^{2}}+\frac{\partial r_{s}}{\partial \tau}\right)+\frac{\partial r_{s-1}}{\partial y}+\frac{\partial F\left(1, y, r_{0}\right)}{\partial r_{0}} r_{s-1}\right. \\
\left.\left.-\frac{\partial^{3} r_{s-2}}{\partial \tau \partial y^{2}}-\frac{\partial^{2} r_{s-2}}{\partial y^{2}}+h_{s}\left(r_{0}, r_{1}, \ldots, r_{s-2}\right)\right]\right\}+0\left(\varepsilon^{n+1}\right) .
\end{gathered}
$$

Here $h_{s}\left(r_{0}, r_{1}, \ldots, r_{s-2}\right)$ denote the known functions expressed by the functions $r_{0}, r_{1}, \ldots, r_{s-2} ; s==2,3, \ldots, n+1$. For example the function $h_{3}\left(r_{0}, r_{1}\right)$ is determined by the formula

$$
h_{3}\left(r_{0}, r_{1}\right)=\frac{1}{2 !} \frac{\partial^{2} F\left(1, y, r_{0}\right)}{\partial r_{0}^{2}} r_{1}^{2} .
$$

Having expanded each function $W_{i}(x, y)=W_{i}(1-\varepsilon \tau, y) ; i=0,1, \ldots, n$ constructed in the first iterative process at the point $(1, y)$ in Taylor formula, writting these expansions in the right hand side of (2.2), we get the following new expansion of the function $W=\sum_{i=0}^{n} \varepsilon^{i} W_{i}(1-\varepsilon \tau, y)$ in new coordinates $(\tau, y)$ with respect to the powers of $\varepsilon$ :

$$
W=\sum_{j=0}^{n+1} \varepsilon^{j} \omega_{j}(\tau, y)+0\left(\varepsilon^{n+2}\right) .
$$

Here $\omega_{0}(\tau, y) \equiv W_{0}(1, y)$ and is independent of $\tau$, the remaining functions $\omega_{k}(\tau, y)$; $k=1,2, \ldots, n+1$ are determined by the formula

$$
\omega_{k}=\sum_{i+j=k}(-1)^{i} \frac{\partial^{i} W_{j}(1, y)}{\partial x^{i}} \tau^{i} ; \quad k=1,2, \ldots, n+1 .
$$


The boundary layer type function near $S_{1}$ is sough in the form

$$
V=\sum_{j=0}^{n+1} \varepsilon^{j} V_{j}(\tau, y)
$$

as a solution of the equation

$$
L_{\varepsilon, 1}(W+V)-L_{\varepsilon, 1} W=0\left(\varepsilon^{n+1}\right) .
$$

From (3.2), (3.3), (3.5),(3.6) we get that the functions $V_{j} ; j=0,1, \ldots, n+1$ should be determined as boundary layer type solutions of the following differential equations:

$$
\begin{gathered}
M V_{0} \equiv \frac{\partial^{3} V_{0}}{\partial \tau^{3}}+\frac{\partial^{2} V_{0}}{\partial \tau^{2}}+\frac{\partial V_{0}}{\partial \tau}=0 \\
M V_{s}=H_{s}\left(\omega_{0}, \omega_{1}, \ldots, \omega_{s-1}, V_{0}, V_{1}, \ldots, V_{s-1}\right) ; s=1,2, \ldots, n+1 .
\end{gathered}
$$

The functions $H_{s}$ are the known functions dependent on the functions $\omega_{0}, \omega_{1}, \ldots, \omega_{s-1}$, $V_{0}, V_{1}, \ldots, V_{s-1}$ and their derivatives. Below we give explicit expressions of the functions $H_{1}$ and $H_{2}$ :

$$
\begin{gathered}
H_{1}\left(\omega_{0}, V_{0}\right)=\frac{\partial V_{0}(\tau, y)}{\partial y}+\frac{\partial F\left(1, y, \omega_{0}+\theta_{1} V_{0}\right)}{\partial u} V_{0}, 0<\theta_{1}<1, \\
H_{2}\left(\omega_{0}, \omega_{1}, V_{0}, V_{1}\right)=\frac{\partial V_{1}}{\partial y}+\frac{\partial F\left(1, y, \omega_{0}+V_{0}\right)}{\partial u}\left(\omega_{1}+V_{1}\right) \\
-\frac{\partial F\left(1, y, \omega_{0}\right)}{\partial u} \omega_{1}+\frac{\partial F\left(1, y, \omega_{0}+V_{0}\right)}{\partial x}(-\tau) \\
-\frac{\partial F\left(1, y, \omega_{0}\right)}{\partial x}(-\tau)+\frac{\partial^{3} V_{0}}{\partial \tau \partial y^{2}}+\frac{\partial^{2} V_{0}}{\partial y^{2}}
\end{gathered}
$$

The boundary conditions for equations (3.7), (3.8) are found from the requirement that the sum $W+V$ satisfies the conditions

$$
\left.(W+V)\right|_{x=1}=0,\left.\frac{\partial}{\partial x}(W+V)\right|_{x=1}=0 .
$$

Having substituted the expressions of $W$ in (2.2), of $V$ in (3.5) in equalities (3.11), and comparing the same powers of $\varepsilon$, we get

$$
\begin{gathered}
\left.V_{i}\right|_{\tau=0}=-\left.W_{i}\right|_{x=1} ; i=0,1, \ldots, n ; V_{n+1}=0, \\
\left.\frac{\partial V_{0}}{\partial \tau}\right|_{\tau=0}=0,\left.\quad \frac{\partial V_{j}}{\partial \tau}\right|_{\tau=0}=\left.\frac{\partial W_{j-1}}{\partial x}\right|_{x=1} ; j=1,2, \ldots, n+1 .
\end{gathered}
$$

Now we construct the functions $V_{0}, V_{1}, \ldots, V_{n+1}$. From (3.7) for $i=0$ from (3.11)(3.13) we get that the function $V_{0}(\tau, y)$ is a boundary layer type solution of equality (3.7) satisfying the following boundary conditions:

$$
\left.V_{0}\right|_{\tau=0}=-W_{0}(1, y),\left.\frac{\partial V_{0}}{\partial \tau}\right|_{\tau=0}=0 .
$$

The characteristic equation corresponding to ordinary differential equation (3.7) has two roots with a negative real part: $\lambda_{1}=-\frac{1}{2}-i \frac{\sqrt{3}}{2}, \lambda_{2}=-\frac{1}{2}+i \frac{\sqrt{3}}{2}$. The number of the 
boundary conditions missing on the boundary $S_{1}$ is also two. This fact shows that problem (1.1)-(1.3) regularly degenerates on the boundary $S_{1}$.

It is clear that the boundary layer type solution of equation (3.7) satisfying boundary conditions (3.14) is determined by the formula

$$
V_{0}(\tau, y)=\frac{W_{0}(1, y)}{\lambda_{1}-\lambda_{2}}\left(\lambda_{2} e^{\lambda_{1} \tau}-\lambda_{1} e^{\lambda_{2} \tau}\right) .
$$

For $s=1$, from (3.8), for $i=1$ from (3.12), for $j=1$ from (3.13) we obtain that (see (3.9)) the function $V_{1}(\tau, y)$ is a boundary layer type solution of the following problem:

$$
\begin{gathered}
\frac{\partial^{3} V_{1}}{\partial \tau^{3}}+\frac{\partial^{2} V_{1}}{\partial \tau^{2}}+\frac{\partial V_{1}}{\partial \tau}=\frac{\partial V_{0}(\tau, y)}{\partial y}+\frac{\partial F\left(1, y, \omega_{0}+\theta_{1} V_{0}\right)}{\partial u} V_{0}, \\
\left.V_{1}\right|_{\tau=0}=-W_{1}(1, y) ;\left.\quad \frac{\partial V_{1}}{\partial \tau}\right|_{\tau=0}=\frac{\partial W_{0}(1, y)}{\partial x} .
\end{gathered}
$$

According to (3.15), the right hand side of equation (3.16) is a boundary layer type function. Therefore, problem (3.16), (3.17) has a boundary layer type solution.

Continuing this process, we construct all the functions $V_{j} ; j=0,1, \ldots, n+1$ entering into the right hand side of equality (3.5). The constructed functions $V_{j}$ are multiplied by the smoothing functions and for the obtained new functions the previous denotations $V_{j}$; $j=0,1, \ldots, n+1$ are preserved. At the expense of smoothing functions for $x=0$ the functions $V_{j} ; j=0,1, \ldots, n+1$ become zero. Hence, from (3.5) and the first boundary condition in (2.16) we obtain that the constructed sum $W+V$ in addition to (3.11) satisfies the boundary condition

$$
\left.(W+V)\right|_{x=0}=0, \quad 0 \leq y \leq 1
$$

as well.

According to Lemma 2.1, as for $x=y$ the functions $W_{i}(x, y) ; i=0,1, \ldots, n$ and in the special case for $x=y=1$ become zero, from the equation (3.8) and boundary conditions (3.12), (3.13) we get that all functions $V_{j}(\tau, y) ; j=0,1, \ldots, n+1$ become zero for $y=0$. Hence, from (3.5) and the second boundary condition in (2.16) it follows that the constructed sum $W+V$ in addition to conditions (3.11), (3.18) satisfies the boundary condition

$$
\left.(W+V)\right|_{y=0}=0,0 \leq x \leq 1
$$

as well.

However, the sum $W+V$ might not satisfy the boundary condition on $S_{2}$. Replace the coordinates near $S_{2}$ by the formulas $x=x, 1-y=\varepsilon t$. Assume that the functions $\varphi_{j}(x, t) ; j=0,1, \ldots, n+1$ are rather smooth functions determined in the domain $[0,1] \times[0,+\infty)$. We accept the following denotation:

$$
\varphi=\sum_{j=0}^{n+1} \varepsilon^{j} \varphi_{j}(x, t)=\varphi_{0}+\delta \varphi .
$$

Here $\delta \varphi$ denotes $\delta \varphi=\varepsilon \varphi_{1}+\varepsilon^{2} \varphi_{2}+\ldots+\varepsilon^{n+1} \varphi_{n+1}$. We take into account the above replacement in the expansion (1.1) of the operator $L_{\varepsilon}$, expand the function $F\left(x, 1-\varepsilon t, \varphi_{0}+\delta \varphi\right)$ at the point $\left(x, 1, \varphi_{0}\right)$ in Taylor formula, and after certain transformations get:

$$
L_{\varepsilon, 2} \varphi \equiv L_{\varepsilon, 2}\left(\sum_{j=0}^{n+1} \varepsilon^{j} \varphi_{j}\right)
$$




$$
\equiv \varepsilon^{-1}\left\{-\left(\frac{\partial^{2} \varphi_{0}}{\partial t^{2}}+\frac{\partial \varphi_{0}}{\partial t}\right)+\sum_{j=1}^{n+1}\left[-\left(\frac{\partial^{2} \varphi_{j}}{\partial t^{2}}+\frac{\partial \varphi_{j}}{\partial t}\right)+g_{j}\left(\varphi_{0}, \varphi_{1}, \ldots, \varphi_{j-1}\right]\right\}+0\left(\varepsilon^{n+1}\right) .\right.
$$

Here $g_{j}\left(\varphi_{0}, \varphi_{1}, \ldots, \varphi_{j-1}\right)$ denote the known functions expressed by the functions $\varphi_{0}, \varphi_{1}, \ldots, \varphi_{j-1} ; j==1,2, \ldots, n+1$. For example, the functions $g_{1}$ and $g_{2}$ are determined by the following formulas:

$$
\begin{gathered}
g_{1}\left(\varphi_{0}\right)=\frac{\partial \varphi_{0}}{\partial x}-\frac{\partial^{3} \varphi_{0}}{\partial x \partial t^{2}}+F\left(x, 1, \varphi_{0}\right) \\
g_{2}\left(\varphi_{1}, \varphi_{0}\right)=\frac{\partial \varphi_{1}}{\partial x}-\frac{\partial^{3} \varphi_{1}}{\partial x \partial t^{2}}+\frac{\partial F\left(x, 1, \varphi_{0}\right)}{\partial \varphi_{0}} \varphi_{1}-\frac{\partial F\left(x, 1, \varphi_{0}\right)}{\partial y} t .
\end{gathered}
$$

We expand every function $W_{i}(x, y)=W_{i}(x, 1-\varepsilon t) ; i=0,1, \ldots, n$ at the point $(x, 1)$ and every function $V_{j}(\tau, y)=V_{j}(\tau, 1-\varepsilon t)$ at the point $(\tau, 1)$ in Taylor formula, and write new expansion of the sum $W+V$ with respect to the powers of $\varepsilon$.

Looking for the boundary layer type function near $S_{2}$ in the form

$$
\eta=\sum_{j=0}^{n+1} \varepsilon^{j} \eta_{j}(x, t)
$$

as the solution of the equation

$$
L_{\varepsilon, 2}(W+V+\eta)-L_{\varepsilon, 2}(W+V)=0\left(\varepsilon^{n+1}\right)
$$

for determine the functions $\eta_{j} ; j=0,1, \ldots, n+1$ we get the following equations:

$$
\begin{gathered}
\frac{\partial^{2} \eta_{0}}{\partial t^{2}}+\frac{\partial \eta_{0}}{\partial t}=0, \\
\frac{\partial^{2} \eta_{s}}{\partial t^{2}}+\frac{\partial \eta_{s}}{\partial t}=P_{s} ; s=1,2, \ldots, n+1 .
\end{gathered}
$$

Here $P_{s}$ denotes $\eta_{0}, \eta_{1}, \ldots, \eta_{s-1}$ and the known functions depedent on the terms in the new expansion of the sum $W+V$. For example, the function $P_{1}$ is determined by the following formula:

$$
P_{1}=\frac{\partial \eta_{0}}{\partial x}+\frac{\partial^{3} \eta_{0}}{\partial x \partial t^{2}}+\frac{\partial F\left(x, 1, W_{0}(x, 1)+V_{0}(\tau, 1)+\theta_{2} \eta_{0}\right)}{\partial u} \eta_{0}, \quad 0<\theta_{2}<1 .
$$

The boundary conditions for equations (3.24), (3.25) are found from the requirement of fulfilment of the sum $W+V+\eta$ the boundary condition

$$
\left.(W+V+\eta)\right|_{y=1}=0 .
$$

From (3.26) the following boundary conditions are obtained:

$$
\left.\eta_{i}\right|_{t=0}=-\left.\left(W_{i}+V_{i}\right)\right|_{y=1} ; i=0,1, \ldots, n ;\left.\quad \eta_{n+1}\right|_{t=0}=-\left.V_{n+1}\right|_{y=1} .
$$

As the functions $V_{j}(\tau, y) ; j=0,1, \ldots, n+1$ become zero for $y=1$, we can write boundary conditions (3.27) as follows:

$$
\left.\eta_{i}\right|_{t=0}=-\left.W_{i}\right|_{y=1} ; i=0,1, \ldots, n ;\left.\quad \eta_{n+1}\right|_{t=0}=0 .
$$

The characteristic equation corresponding to ordinary differential equations (3.24), (3.25) has one negative root. One boundary condition on $S_{2}$ misses. So, problem (1.1)-(1.3) regularly degenerate on the boundary $S_{2}$ as well. 
For $i=0$, from (3.28) it follows that the function $\eta_{0}(x, t)$ is a boundary layer type solution of equation (3.24) satisfying the boundary condition

$$
\left.\eta_{0}\right|_{t=0}=-W_{0}(x, 1) .
$$

It is clear that the function $\eta_{0}(x, t)$ is determined by the formula

$$
\eta_{0}(x, t)=-W_{0}(x, 1) e^{-t} .
$$

Continuing the process, the functions $\eta_{1}, \eta_{2}, \ldots, \eta_{n+1}$ are determined as boundary layer type solutions of equations (3.25) satisfying appropriate boundary conditions in (3.28). All constructed functions $\eta_{j}$ are multiplied by the smoothing functions and for the newly obtained functions the previous denotations $\eta_{j} ; j=0,1, \ldots, n+1$ are preserved. As for $y=0$ the functions $\eta_{j} ; j=0,1, \ldots, n+1$ become zero at the expense of the smooth functions, hence from (3.19) and (3.22) it follows that the sum $W+V+\eta$ in addition to condition (3.26) satisfies the boundary condition

$$
\left.(W+V+\eta)\right|_{y=0}=0
$$

as well.

According to Lemma 2.1, as all the functions $\eta_{j} ; j=0,1, \ldots, n+1$ become zero together with derivatives for $x=1$, by (3.22) the condition $\left.\eta\right|_{x=1}=0$ is satisfied. Hence and from (3.11) we get that the sum $W+V+\eta$ in addition to conditions (3.26), (3.30) satisfies the boundary conditions

$$
\left.(W+V+\eta)\right|_{x=1}=0,\left.\quad \frac{\partial}{\partial x}(W+V+\eta)\right|_{x=1}=0
$$

as well.

Assume that when the function $F(x, y, u)$ is linearly dependent on the variable $u$, satisfies the condition

$$
\frac{\partial^{k} f(0,1)}{\partial x^{k_{1}} \partial y^{k_{2}}}=0 ; k=k_{1}+k_{2} ; k=0,1, \ldots, 2 n+3,
$$

when is linearly independent on the variable $u$, satisfies the condition

$$
\frac{\partial^{k} F(0,1,0)}{\partial x^{k_{1}} \partial y^{k_{2}} \partial u^{k_{3}}}=0 ; k=k_{1}+k_{2}+k_{3} ; k=0,1, \ldots, 2 n+3 .
$$

Then all the functions $\eta_{j} ; j=0,1, \ldots, n+1$ will become zero for $x=0$. Hence and from (3.18) we get that the sum $W+V+\eta$ in addition to conditions $(3,26),(3.30),(3.31)$ satisfies the boundary condition

$$
\left.(W+V+\eta)\right|_{x=0}=0
$$

as well.

Thus, we constructed such a function $\tilde{u}=W+V+\eta$ that according to (3.26), (3.30), (3.31), (3.34) this function satisfies the following boundary conditions:

$$
\begin{gathered}
\left.\tilde{u}\right|_{x=0}=\left.\tilde{u}\right|_{x=1}=0,\left.\quad \frac{\partial \tilde{u}}{\partial x}\right|_{x=1}=0, \quad 0 \leq y \leq 1 \\
\left.\tilde{u}\right|_{y=0}=\left.\tilde{u}\right|_{y=1}=0, \quad 0 \leq x \leq 1 .
\end{gathered}
$$


Summing the equalities (2.1), (3.6), (3.23) side by side, we get that the function $\tilde{u}$ is the solution of the following equation:

$$
L_{\varepsilon} \tilde{u}=0\left(\varepsilon^{n+1}\right) .
$$

We denote the difference between the exact solution $u$ and $\tilde{u}$ by of problem (1)-(3) $z$ :

$$
u-\tilde{u}=z .
$$

$z$ is said to be a remainder term.

\section{Estimating the remainder term and the main result}

From (3.38) we obtain that the asymptotic expansion of the solution of problem (1.1)-(1.3) with respect to a small parameter is as follows:

$$
u=\sum_{i=0}^{n} \varepsilon^{i} W_{i}+\sum_{j=0}^{n+1} \varepsilon^{j} V_{j}+\sum_{j=0}^{n+1} \varepsilon^{j} \eta_{j}+z .
$$

We now estimate the remainder term. Acting on the both hand sides of equality (3.38) by the operator $L_{\varepsilon}$ and boundary conditions (1.2), (1.3), we obtain that the function $z$ is the solution of the following boundary value problem:

$$
\begin{gathered}
L_{\varepsilon} z=0\left(\varepsilon^{n+1}\right), \\
\left.z\right|_{x=0}=\left.z\right|_{x=1}=0,\left.\quad \frac{\partial z}{\partial x}\right|_{x=1}=0, \quad 0 \leq y \leq 1, \\
\left.z\right|_{y=0}=\left.z\right|_{y=1}=0, \quad 0 \leq x \leq 1 .
\end{gathered}
$$

The following proposition is valid.

Lemma 4.1 The following estimation is true for the function $z$ being the solution of boundary value problem (4.2)-(4.4).

$$
\begin{gathered}
\varepsilon^{2} \int_{0}^{1}\left(\left.\frac{\partial z}{\partial x}\right|_{x=0}\right)^{2} d y+\varepsilon \iint_{D}\left[\left(\frac{\partial z}{\partial x}\right)^{2}+\left(\frac{\partial z}{\partial y}\right)^{2}\right] d x d y+ \\
+c_{1} \iint_{D} z^{2} d x d y \leq c_{2} \varepsilon^{2(n+1)}
\end{gathered}
$$

Here the constants $c_{1}>0, c_{2}>0$ are independent of $\varepsilon$.

For proving Lemma 4.1 we scalarly multiply the both hand sides of equation (4.2) by $z$ and using boundary conditions (4.3), (4.4) we piecewise integrate the obtained terms in domain $D$.

The obtained results may be generalized in the form of the following theorem.

Theorem 4.1 Assume that the function $F(x, y, u)$ is included in space $C^{2 n+3}(D \times(-\infty,+\infty))$, when this function is linearly dependent on the variable u satisfies conditions (2.10), (3.32), when is linearly independent of the variable u the conditions (2.11), (2.12), (3.33). Then the asymptotic expansion of the solution of problem (1.1)-(1.3) with respect to a small parameter is in the form (4.1). Here the $W_{i}$-functions are determined in the first iterative process, $V_{j}, \eta_{j}$-functions are boundary layer type functions, respectively near $x=1$ and $y=1$ and are determined by means of the appropriate iterative processes, $z$ is a remainder term, the estimation (4.5) is valid for it. 


\section{References}

1. Vishik, M.I., Lusternik, L.A.: Regular degeneration and boundary layer for linear differential equations with a small parameter, UMN, vol. 12, 5 (77), 3-122 (1957) (in Russian).

2. Vishik, M.I., Lusternik, L.A.: Solution of some perturbation problems in the case of matrices and self-adjoint, not self-adjoint differential equations, UMN, vol. 15, 3 (93), 3-80 (1960) (in Russian).

3. M.M. Sabzaliev, M.E. Kerimova: Asymptotics of the solution in a rectangle of a boundary value problem for one-characteristic differential equation degenerating into a parabolic equation, Trans. Natl. Acad. Sci. Azerb. Ser. Phys.-Tech. Math. Sci. 34 (4) (2014), Mathematics and Mechanics, 97-106 (2014)

4. M.M. Sabzaliev, M.E. Kerimova: Asymptotics of the solution of a boundary value problem for one-characteristic differential equation degenerating into a parabolic equation in an infinite strip, Nonlinear Analysis and Differential Equations, 2 (3), 125-133 (2014)

5. M.M. Sabzaliev, M.E. Kerimova: Asymptotics of the solution of a boundary value problem in an infinite semi-strip for one-characteristic differential equation degenerating into a parabolic equation, Nonlinear Analysis and Differential Equations, 4 (4), 179187 (2016)

6. M.M. Sabzaliev: The asymptotic form of the solution of boundary value problem for quasilinear elliptic equation in the rectangular domain, Trans. Natl. Acad. Sci. Azerb. Ser. Phys.-Tech. Math. Sci. 25 (7), Math. Mech., 107-118 (2005) 